\title{
Nonlinear Inequalities with Double Riesz Potentials
}

\author{
Marius Ghergu $^{1,2} \cdot{\text { Zeng } \text { Liu }^{3} \cdot \text { Yasuhito Miyamoto }^{4} \text { (D) } \cdot \text { Vitaly Moroz }}^{5}$
}

Received: 8 June 2021 / Accepted: 7 October 2021 / Published online: 26 October 2021

(C) The Author(s) 2021

\begin{abstract}
We investigate the nonnegative solutions to the nonlinear integral inequality $u \geq I_{\alpha} *\left(\left(I_{\beta} *\right.\right.$ $\left.\left.u^{p}\right) u^{q}\right)$ a.e. in $\mathbb{R}^{N}$, where $\alpha, \beta \in(0, N), p, q>0$ and $I_{\alpha}, I_{\beta}$ denote the Riesz potentials of order $\alpha$ and $\beta$ respectively. Our approach relies on a nonlocal positivity principle which allows us to derive optimal ranges for the parameters $\alpha, \beta, p$ and $q$ to describe the existence and the nonexistence of a solution. The optimal decay at infinity for such solutions is also discussed.
\end{abstract}

Keywords Nonlinear integral inequalities $\cdot$ Riesz potentials $\cdot$ Nonlocal positivity principle $\cdot$ Liouville theorems

Mathematics Subject Classification (2010) Primary 45G10; Secondary 31B10 - 45M05

ZL was supported by NSFC Grant Numbers $11901418,11771319$.

YM was supported by JSPS KAKENHI Grant Numbers 19H01797, 19 H05599.

Vitaly Moroz

v.moroz@swansea.ac.uk

Marius Ghergu

marius.ghergu@ucd.ie

Zeng Liu

zliu@mail.usts.edu.cn

Yasuhito Miyamoto

miyamoto@ms.u-tokyo.ac.jp

1 School of Mathematics and Statistics, University College Dublin, Belfield, Dublin 4, Ireland

2 Institute of Mathematics Simion Stoilow of the Romanian Academy, 21 Calea Grivitei St., 010702 Bucharest, Romania

3 Department of Mathematics, Suzhou University of Science and Technology, Suzhou, 215009, People's Republic of China

4 Graduate School of Mathematical Sciences, The University of Tokyo, 3-8-1 Komaba, Meguro-ku, Tokyo 153-8914, Japan

5 Mathematics Department, Swansea University, Bay Campus, Fabian Way, Swansea SA1 8EN, Wales, United Kingdom 


\section{Introduction}

We study nonnegative solutions of the following integral inequality with double Riesz potentials

$$
u \geq I_{\alpha} *\left(\left(I_{\beta} * u^{p}\right) u^{q}\right) \text { a.e. in } \mathbb{R}^{N},
$$

in the range $N \geq 2, p, q>0, \alpha, \beta \in(0, N)$ and $I_{\gamma}(x):=A_{\gamma}|x|^{-(N-\gamma)}$ is the Riesz potential of order $\gamma \in(0, N)$ where $*$ denotes the standard convolution in $\mathbb{R}^{N}$. The choice of the normalisation constant $A_{\gamma}:=\frac{\Gamma((N-\gamma) / 2)}{\pi^{N / 2} 2^{\gamma} \Gamma(\gamma / 2)}$ ensures that $I_{\gamma}(x)$ could be interpreted as the Green function of $(-\Delta)^{\gamma / 2}$ in $\mathbb{R}^{N}$, and that the semigroup property $I_{\alpha+\beta}=I_{\alpha} * I_{\beta}$ holds for all $\alpha, \beta \in(0, N)$ such that $\alpha+\beta<N$, see for example [9, p. 45].

By a nonnegative solution of Eq. 1 we understand a function $u \in L_{l o c}^{1}\left(\mathbb{R}^{N}\right), u \geq 0$, such that the right hand side of Eq. 1 is well-defined, i.e.

$$
I_{\alpha} *\left(\left(I_{\beta} * u^{p}\right) u^{q}\right)<+\infty \text { a.e. in } \mathbb{R}^{N}
$$

and the inequality Eq. 1 holds a.e. in $\mathbb{R}^{N}$. Condition Eq. 2 above is equivalent (see Lemma 2.1 below) to

$$
\left(I_{\beta} * u^{p}\right) u^{q} \in L^{1}\left((1+|x|)^{-(N-\alpha)} d x, \mathbb{R}^{N}\right) .
$$

Integral inequalities and equations featuring a single Riesz potential have been extensively investigated in the past two decades. The prototype model

$$
u=I_{\alpha} * u^{p} \quad \text { in } \quad \mathbb{R}^{N},
$$

has been largely investigated starting with the seminal works in [3, 4]. More recently, various techniques have been devised to deal with systems of equations or inequalities that incorporate anisotropic or more general potentials [2, 8, 11, 12, 19, 20].

Our aim in this paper is to provide an optimal description for the existence and nonexistence of positive solutions to the integral inequality (1).

For any $\alpha>0$, the fractional Laplacian $(-\Delta)^{\alpha / 2}$ is defined by means of the Fourier transform

$$
\widehat{(-\Delta)^{\alpha / 2}} u(\xi):=|\xi|^{\alpha} \hat{u}(\xi)
$$

for all $u \in \mathcal{S}^{\prime}$ such that $|\xi|^{\alpha} u \in \mathcal{S}^{\prime}$, here $\mathcal{S}^{\prime}$ stands for the space of tempered distributions on $\mathbb{R}^{N}$ which is the dual of the Schwartz space $\mathcal{S}$.

Since for $\alpha \in(0, N)$ the Riesz potential $I_{\alpha}$ can be interpreted as the inverse of $(-\Delta)^{\alpha / 2}$ (cf. [18]*Sect.5.1 or [2, Section 2.1]), under some extra integrability conditions on $u \geq 0$, inequality (1) is equivalent to the elliptic inequality

$$
(-\Delta)^{\alpha / 2} u \geq\left(I_{\beta} * u^{p}\right) u^{q} \quad \text { a.e. in } \mathbb{R}^{N},
$$

provided that both Eqs. 1 and 4 are well-defined. This is the case, for instance, if Eq. 3 holds and $u$ belongs to the homogeneous Sobolev space $\dot{H}^{\alpha / 2}\left(\mathbb{R}^{N}\right)$, so that Eq. 4 is understood in the weak sense. Pointwise interpretations of the inequality Eq. 4 for non-integer $\alpha / 2$ are also possible, cf. [2, Theorem 2.13]. For a comparison of different definitions of the higher order fractional Laplacian $(-\Delta)^{\alpha / 2}$ see [1].

Inequality (4) is a Choquard type inequality. Equations and inequalities of such structure originate from mathematical physics and have attracted considerable interest of mathematicians in the past decades. Precisely, the equation

$$
-\Delta u+u=\left(I_{2} * u^{2}\right) u \quad \text { in } \mathbb{R}^{3}
$$

was introduced by Fröhlich [5, 6] and Pekar [17] as a model for polaron, where free electrons in an ionic lattice interact with phonons associated to deformations of the lattice or 
with the polarisation that it creates on the medium. The same equation was proposed by Choquard [13] in the modelling of a one-component plasma. Closely related to the Eq. 5 is the Hartree equation

$$
i \partial_{t} u=\Delta u+\left(I_{2} * u^{2}\right) u \quad(x, t) \in \mathbb{R}^{3} \times(0, \infty) .
$$

Indeed, standing waves of Eq. 6 are solutions to Eq. 5. Further, steady state solutions to the Schrödinger equation

$$
i \partial_{t} u=\Delta u+\left(I_{\beta} * u^{p}\right) u^{q} \quad(x, t) \in \mathbb{R}^{N} \times(0, \infty),
$$

satisfy Eq. 4 with $\alpha=2$. More recently, the Choquard Eq. 5 appears with the name of Schrödinger-Newton equation in models coupling the Schrödinger equation of quantum physics together with nonrelativistic Newtonian gravity. For a mathematical account on this topic we refer the reader to the survey work [16]. In the second order elliptic case $\alpha=2$ optimal regimes for the existence and nonexistence of positive solutions to inequality Eq. 4 were fully investigated in [15]. The higher-order polyharmonic case $\alpha / 2=m \in \mathbb{N}$ was recently studied in [7], where (amongst other results) optimal existence and nonexistence regimes for the Eq. 4 were obtained for the exponents $p \geq 1$ and $q>1$, see [7, Theorem $1.4]$.

In this work we extend earlier results in [15] and [7] to the full admissible range $\alpha \in$ $(0, N)$ and exponents $p, q>0$. Our approach is different from the techniques in [7], which were based on the poly-superharmonic properties of $(-\Delta)^{m}$ in the elliptic framework of Eq. 4. Instead, we work entirely with the double-nonlocal inequality Eq. 1. Our analysis of Eq. 1 employs only direct Riesz kernel estimates, and a new version of the nonlocal positivity principle in Lemma 3.1, inspired by [15, Proposition 3.2]. This has the advantage of incorporating the fractional case of noninteger $\alpha / 2$ in a seemingly effortless way, and does not rely on comparison principles or Harnack type inequalities, which are commonly used for similar Liouville type results in the elliptic framework, but which are generally speaking not available in the case of the higher-order fractional Laplacians $(-\Delta)^{\alpha / 2}$ with $\alpha>2$.

The main result of this work related to the existence of positive solutions to Eq. 1 reads as follows.

Theorem 1.1 Let $p, q>0$. Then, inequality (1) has a nontrivial nonnegative solution $u \in L_{l o c}^{1}\left(\mathbb{R}^{N}\right)$ which satisfies (2) if and only if

$$
\begin{cases}p>\frac{\beta}{N-\alpha} & \\ p+q>\frac{N+\beta}{N-\alpha} & \text { if } \beta>N-\alpha, \\ q>\frac{\beta}{N-\alpha} & \text { if } \beta=N-\alpha, \\ q \geq 1 & \text { if } \beta<N-\alpha . \\ q>1-\frac{N-\alpha-\beta}{N} p & \end{cases}
$$

The necessary part of the proof follows directly from Propositions 2.3, 4.1-4.5 below. The sufficiency follows from Propositions 5.4-5.7, where we construct explicitly smooth positive radial solutions to Eq. 1 . In the case $\alpha=2$ our results are fully consistent with the results established in [15, Theorem 1] for the 2 nd order elliptic inequality (4). The 
nonexistence of positive solutions to double-nonlocal inequality Eq. 1 with $p>1, q>0$ and $p+q \leq \frac{N+\beta}{N-\alpha}$ was established by different methods in [10, Theorem 1].

Remark 1.2 In Section 5 we also discuss the optimal decay of solutions to Eq. 1 in terms of the parameters $\alpha, \beta, p$ and $q$. Clearly (see Eq. 11), if $u \geq 0$ is a nontrivial solution of Eq. 1 then $\liminf _{|x| \rightarrow \infty} u(x)|x|^{N-\alpha}>0$. In particular, for $R \gg 1$ we have

$$
\int_{B_{2 R} \backslash B_{R}} u \geq c R^{\alpha} .
$$

In Proposition 5.1 we establish an integral lower bound

$$
\int_{B_{2 R} \backslash B_{R}} u \geq c R^{\frac{\alpha+\beta-N q}{1-q}},
$$

which is stronger than Eq. 7 when $\alpha+\beta<N$ and $q<\frac{\beta}{N-\alpha}<1$. In Propositions 5.4 and 5.1 we construct positive radial solutions $u$ to Eq. 1 that confirm the optimality of Eq. 7 when $q>\frac{\beta}{N-\alpha}$ and of Eq. 8 when $q<\frac{\beta}{N-\alpha}$. When $q=\frac{\beta}{N-\alpha}$ the bounds in Eqs. 7 and 8 coincide. In that case in Propositions 5.6 and 5.7 we construct positive radial solutions to Eq. 1 that satisfy Eq. 7 up to a $\log (q<1)$ or arbitrary small polynomial $(q=1)$ corrections. In the case $\alpha=2$ such corrections are necessary, see [15, Proposition 4.12, 4.13].

\section{Preliminaries}

In this section we collect some useful facts for our approach.

Lemma 2.1 Let $f: \mathbb{R}^{N} \rightarrow \mathbb{R}$ be a nonnegative measurable function. Then, the Riesz potential $I_{\alpha} * f$ of order $\alpha \in(0, N)$ is well defined, in the sense that

$$
I_{\alpha} * f<+\infty \text { a.e. in } \mathbb{R}^{N},
$$

if and only if

$$
f \in L^{1}\left((1+|x|)^{-(N-\alpha)} d x, \mathbb{R}^{N}\right) .
$$

Moreover, if Eq. 9 fails then $I_{\alpha} * f=+\infty$ everywhere in $\mathbb{R}^{N}$, see [9]*p.61-62. We present the proof of the lemma for completeness.

Proof Assume first that Eq. 9 holds. Then, for any $x, y \in \mathbb{R}^{N}, x \neq 0$ we have $|x-y|^{N-\alpha} \leq c\left(|x|^{N-\alpha}+|y|^{N-\alpha}\right) \leq c \max \left\{1,|x|^{N-\alpha}\right\}\left(1+|y|^{N-\alpha}\right) \leq c \max \left\{1,|x|^{N-\alpha}\right\}(1+|y|)^{N-\alpha}$. Thus, for any $x \in \mathbb{R}^{N} \backslash\{0\}$ such that Eq. 9 holds, we have

$$
\infty>\left(I_{\alpha} * f\right)(x) \geq \frac{1}{c \max \left\{1,|x|^{N-\alpha}\right\}} \int_{\mathbb{R}^{N}} \frac{f(y) d y}{(1+|y|)^{N-\alpha}},
$$

which yields Eq. 10.

Conversely, assume now that Eq. 10 holds. For any $R>0$ we have

$$
\int_{B_{R}} f(y) d y \leq(1+R)^{N-\alpha} \int_{B_{R}} \frac{f(y) d y}{(1+|y|)^{N-\alpha}} \leq(1+R)^{N-\alpha} \int_{\mathbb{R}^{N}} \frac{f(y) d y}{(1+|y|)^{N-\alpha}}<\infty .
$$


This shows that $f \in L_{l o c}^{1}\left(\mathbb{R}^{N}\right)$. If $x \in \mathbb{R}^{N} \backslash\{0\}$, then

$$
\begin{aligned}
\left(I_{\alpha} * f\right)(x) & =\int_{|y| \leq 2|x|} \frac{f(y) d y}{|x-y|^{N-\alpha}}+\int_{|y|>2|x|} \frac{f(y) d y}{|x-y|^{N-\alpha}} \\
& \leq \int_{|y| \leq 2|x|} \frac{f(y) d y}{|x-y|^{N-\alpha}}+2^{N-\alpha} \int_{|y|>2|x|} \frac{f(y) d y}{|y|^{N-\alpha}} \\
& <\infty .
\end{aligned}
$$

In the same spirit to the above proof, if $f \geq 0$ and Eq. 9 (or, equivalently Eq. 10) holds, then

$$
I_{\alpha} * f(x) \geq \frac{c}{|x|^{N-\alpha}} \int_{B_{|x|}(0)} f(y) d y .
$$

One of the elementary yet important for our approach consequences of Eq. 11 is the following estimate, which we will be using frequently, and which to some extent is the counterpart of the Harnack inequalities on the annuli in the study of Eq. 4.

Lemma 2.2 Let $\alpha \in(0, N), \theta>0$ and $0 \leq f \in L^{1}\left((1+|x|)^{-(N-\alpha)} d x, \mathbb{R}^{N}\right)$. Then for all $R>0$ we have

$$
\int_{B_{2 R} \backslash B_{R}}\left(I_{\alpha} * f\right)^{\theta} \geq C R^{N-(N-\alpha) \theta}\left(\int_{B_{R}} f\right)^{\theta} .
$$

Proof Follows from Eq. 11 by integration.

An obvious implication of Eq. 11 is that $I_{\alpha} * f$ can not decay faster than $I_{\alpha}$ at infinity, even if the function $f$ is compactly supported. Recall also that if $f \geq 0$ then an elementary estimate shows that for every $x \in \mathbb{R}^{N}$,

$$
I_{\alpha} * f(x) \geq \frac{A_{\alpha}}{R^{N-\alpha}} \int_{B_{R}(x)} f(y) d y .
$$

As a consequence, if $u \geq 0$ is a solution of Eq. 1 that is positive on a set of positive measure, then $u$ is everywhere strictly positive on $\mathbb{R}^{N}$ and the following lower bounds must hold:

$$
\begin{aligned}
u(x) & \geq c(1+|x|)^{-(N-\alpha)}, \\
I_{\beta} * u^{p}(x) & \geq c(1+|x|)^{-(N-\beta)} .
\end{aligned}
$$

On the other hand, Eq. 2 requires

$$
\begin{aligned}
u^{p} & \in L^{1}\left((1+|x|)^{-(N-\beta)} d x, \mathbb{R}^{N}\right), \\
\left(I_{\beta} * u^{p}\right) u^{q} & \in L^{1}\left((1+|x|)^{-(N-\alpha)} d x, \mathbb{R}^{N}\right) .
\end{aligned}
$$

Combining the competing upper and lower bounds immediately leads to the following nonexistence result.

Proposition 2.3 Let $p, q>0$ and assume that either $p \leq \frac{\beta}{N-\alpha}$, or $\alpha+\beta>N$ and $q \leq \frac{\beta}{N-\alpha}-1$. If $u \geq 0$ is a solution of Eq. 1 then $u \equiv 0$. 
Proof First we note that Eqs. 14 and 16 are incompatible when $p \leq \frac{\beta}{N-\alpha}$. Then we observe that Eqs. 14, 15 and 17 are incompatible when $0<q \leq \frac{\beta}{N-\alpha}-1$.

Remark 2.4 We will see in Proposition 4.3 below that $q \leq \frac{\beta}{N-\alpha}-1$ is suboptimal for the nonexistence and could be refined.

\section{Nonlocal Positivity Principle}

The nonexistence result in Proposition 2.3 "decouples" the values of $p$ and $q$. In order to deduce an estimate which involves the quantity $p+q$ which appears in Theorem 1.1, we need the following lemma, inspired by [14, Proposition 2.1] and [15]*Section 3.

Lemma 3.1 (Nonlocal positivity principle) Let $\alpha \in(0, N)$ and $V: \mathbb{R}^{N} \rightarrow[0, \infty)$ be a measurable function. Assume that there exists $u \in L_{\text {loc }}^{1}\left(\mathbb{R}^{N}\right)$ such that $u>0$ a.e. in $\mathbb{R}^{N}$, $V u \in L^{1}\left((1+|x|)^{-(N-\alpha)} d x, \mathbb{R}^{N}\right)$ and

$$
u \geq I_{\alpha} *(V u) \text { a.e. in } \mathbb{R}^{N} .
$$

Then for every $R>0$ and $0 \leq \varphi \in C_{c}^{\infty}\left(B_{R}\right)$,

$$
\int_{B_{R}} \varphi^{2} \geq C R^{\alpha-N}\left(\int_{B_{R}} \sqrt{V} \varphi\right)^{2} .
$$

Proof Take $\psi:=\frac{\varphi^{2}}{u}$ as a test function in Eq. 18. Then

$$
\begin{aligned}
\int_{B_{R}} \varphi^{2} \geq & \iint_{B_{R} \times B_{R}} I_{\alpha}(x-y) V(y) u(y) \frac{\varphi^{2}(x)}{u(x)} d y d x \\
= & \frac{1}{2} \iint_{B_{R} \times B_{R}} I_{\alpha}(x-y)\left(V(x) u(x) \frac{\varphi^{2}(y)}{u(y)}+V(y) u(y) \frac{\varphi^{2}(x)}{u(x)}\right) d x d y \\
= & \iint_{B_{R} \times B_{R}} I_{\alpha}(x-y) \sqrt{V(x) V(y)} \varphi(y) \varphi(x) d x d y \\
& +\frac{1}{2} \iint_{B_{R} \times B_{R}} I_{\alpha}(x-y) u(x) u(y)\left(\sqrt{V(x)} \frac{\varphi(y)}{u(y)}-\sqrt{V(y)} \frac{\varphi(x)}{u(x)}\right)^{2} d x d y \\
\geq & \frac{A_{\alpha}}{2^{N-\alpha} R^{N-\alpha}}\left(\int_{B_{R}} \sqrt{V(x)} \varphi(x) d x\right)^{2},
\end{aligned}
$$

since

$$
I_{\alpha}(x-y) \geq \frac{A_{\alpha}}{2^{N-\alpha} R^{N-\alpha}} \quad\left(x, y \in B_{R}\right),
$$

which completes the proof.

Remark 3.2 Nonlocal inequality (18) can be interpreted as the "inversion" of the fractional Schrödinger inequality

$$
(-\Delta)^{\alpha / 2} u-V u \geq 0 \quad \text { in } \quad \mathbb{R}^{N} .
$$

In this context Lemma 3.1 can be seen as a higher-order version of the fractional AgmonAllegretto-Piepenbrink's positivity principle: if Eq. 18 has a positive supersolution then a certain variational inequality which involves the potential $V$ must hold. We will see that 
Lemma 3.1 alongside with the standard integral estimate Eq. 12 of the Riesz potentials are sufficient for the complete analysis of the existence and nonexistence of positive solutions of the nonlinear inequality Eq. 1.

Using Lemma 3.1 we establish the following estimate.

Proposition 3.3 Let $p, q>0$ and $u>0$ be a solution of Eq. 1. Then, for every $R>0$ and every $\varphi \in C_{c}^{\infty}\left(B_{R}\right)$,

$$
\int_{B_{R}} \varphi^{2} \geq C R^{\alpha+\beta-2 N}\left(\int_{B_{R}} u^{p}\right)\left(\int_{B_{R}} u^{\frac{q-1}{2}} \varphi\right)^{2} .
$$

Proof For every $\varphi \in C_{c}^{\infty}\left(B_{R}\right)$, by Lemma 3.1 with $V=\left(I_{\beta} * u^{p}\right) u^{q-1}$, and using a similar inequality to Eq. 20 for $I_{\beta} * u^{p}$, we obtain

$$
\begin{aligned}
\int_{B_{R}} \varphi^{2} & \geq C R^{\alpha-N}\left(\int_{B_{R}}\left(\left(I_{\beta} * u^{p}\right) u^{q-1}\right)^{1 / 2} \varphi\right)^{2} \\
& \geq C^{\prime} R^{\alpha+\beta-2 N}\left(\int_{B_{R}} u^{p}\right)\left(\int_{B_{R}} u^{\frac{q-1}{2}} \varphi\right)^{2} .
\end{aligned}
$$

One of the principal tools in the subsequent analysis is the following decay estimate on the solutions of Eq. 1, which is an adaptation of Eq. 21. Note that for $q<1$ our estimate contains a lower bound on the solution, since the 2 nd integral involves a negative power of $u$.

Corollary 3.4 Let $p, q>0$ and $u>0$ be a solution of Eq. 1. Then for every $R>0$,

$$
\left(\int_{B_{2 R}} u^{p}\right)\left(\int_{B_{2 R} \backslash B_{R}} u^{\frac{q-1}{2}}\right)^{2} \leq C R^{3 N-\alpha-\beta} .
$$

Proof Take $\varphi_{R}(x)=\varphi(x / R)$, where $\varphi \in C_{c}^{\infty}\left(\mathbb{R}^{N}\right)$ is such that $\operatorname{supp}(\varphi) \subset \bar{B}_{4} \backslash B_{1 / 2}$, $\varphi \equiv 1$ on $\bar{B}_{2} \backslash B_{1}$ and $0 \leq \varphi \leq 1$. Then,by Eq. 21 we find

$$
\begin{aligned}
c R^{N}=\int_{B_{4 R}} \varphi_{R}^{2} & \geq C^{\prime} R^{\alpha+\beta-2 N}\left(\int_{B_{4 R}} u^{p}\right)\left(\int_{B_{4 R}} u^{\frac{q-1}{2}} \varphi_{R}\right)^{2} \\
& \geq C^{\prime} R^{\alpha+\beta-2 N}\left(\int_{B_{2 R}} u^{p}\right)\left(\int_{B_{2 R} \backslash B_{R}} u^{\frac{q-1}{2}}\right)^{2} .
\end{aligned}
$$

\section{Nonexistence}

In this section we derive several nonexistence results for Eq. 1. Our approach is inspired by [15] which studied the inequality Eq. 4 in the semilinear the case $\alpha=2$, yet with substantial modifications. In particular, in this work we completely avoid the use of the comparison principle and Harnack's inequalities, which are not applicable in the framework of the double-nonlocal inequality Eq. 1. It turns out that Harnack inequality estimates in the context of Eq. 1 can be replaced by the estimate Eq. 12 . 
Proposition 4.1 Let $p, q>0$ and assume that $p+q<1$. If $u \geq 0$ is a solution of Eq. 1 then $u \equiv 0$.

Proof Since $u^{p} \in L^{1}\left((1+|x|)^{-(N-\beta)} d x\right)$ and $\beta<N$, by Lebesgue's dominated convergence theorem

$$
\int_{B_{2 R} \backslash B_{R}} u^{p}=o\left(R^{N-\beta}\right)=o\left(R^{N}\right) \quad \text { as } R \rightarrow \infty .
$$

Since $p+q<1$, we may apply Hölder's inequality and then the estimate Eq. 22 to obtain

$$
\begin{aligned}
c R^{N}=\int_{B_{2 R} \backslash B_{R}} 1 & =\int_{B_{2 R} \backslash B_{R}} u^{\frac{p(1-q)}{2 p+1-q}} u^{\frac{(q-1) p}{2 p+1-q}} \\
& \leq\left(\int_{B_{2 R} \backslash B_{R}} u^{p}\right)^{\frac{1-q}{2 p+1-q}}\left(\int_{B_{2 R} \backslash B_{R}} u^{\frac{q-1}{2}}\right)^{\frac{2 p}{2 p+1-q}} \\
& \leq\left(\int_{B_{2 R} \backslash B_{R}} u^{p}\right)^{\frac{1-q-p}{2 p+1-q}}\left[\left(\int_{B_{2 R} \backslash B_{R}} u^{p}\right)\left(\int_{B_{2 R} \backslash B_{R}} u^{\frac{q-1}{2}}\right)^{2}\right]^{\frac{p}{2 p+1-q}} \\
& \leq\left(\int_{B_{2 R} \backslash B_{R}} u^{p}\right)^{\frac{1-q-p}{2 p+1-q}}\left(C R^{3 N-\alpha-\beta}\right)^{\frac{p}{2 p+1-q}} \\
& \leq o(1) R^{N \frac{1-q-p}{2 p+1-q}}\left(R^{3 N}\right)^{\frac{p}{2 p+1-q}} \\
& =o(1) R^{N},
\end{aligned}
$$

which raises a contradiction.

Proposition 4.2 Let $p, q>0$ and assume that $1 \leq p+q \leq \frac{N+\beta}{N-\alpha}$. If $u \geq 0$ is a solution of Eq. 1 then $u \equiv 0$.

Proof Assume first $p+q<\frac{N+\beta}{N-\alpha}$. By the Cauchy-Schwarz inequality,

$$
\int_{B_{2 R}} u^{p} \geq c R^{-N}\left(\int_{B_{2 R}} u^{\frac{p}{2}}\right)^{2},
$$

and so Eq. 22 implies

$$
C R^{4 N-\alpha-\beta} \geq\left(\int_{B_{2 R}} u^{\frac{p}{2}}\right)^{2}\left(\int_{B_{2 R} \backslash B_{R}} u^{\frac{q-1}{2}}\right)^{2} .
$$

Using Eq. 23 and the Cauchy-Schwarz again together with $u \geq c|x|^{-(N-\alpha)}$ in $\mathbb{R}^{N} \backslash B_{1}$ (that follows from Eq. 14), we obtain

$$
\begin{aligned}
C R^{4 N-\alpha-\beta} & \geq\left(\int_{B_{2 R}} u^{\frac{p}{2}}\right)^{2}\left(\int_{B_{2 R} \backslash B_{R}} u^{\frac{q-1}{2}}\right)^{2} \\
& \geq\left(\int_{B_{2 R} \backslash B_{R}} u^{\frac{p+q-1}{4}}\right)^{4} \\
& \geq c R^{4 N-(N-\alpha)(p+q-1)}
\end{aligned}
$$

which is a contradiction since $0 \leq p+q-1<\frac{\alpha+\beta}{N-\alpha}$. 
Assume now $p+q=\frac{N+\beta}{N-\alpha}$. By Hölder's inequality we find

$$
\begin{aligned}
\left(\int_{\mathbb{R}^{N}}\left(I_{\beta} * u^{p}\right) u^{q}\right)^{2} & =\left(\iint_{\mathbb{R}^{N} \times \mathbb{R}^{N}} I_{\beta}(x-y) u^{p}(y) u^{q}(x)\right)\left(\iint_{\mathbb{R}^{N} \times \mathbb{R}^{N}} I_{\beta}(x-y) u^{p}(x) u^{q}(y)\right) \\
& \geq\left(\iint_{\mathbb{R}^{N} \times \mathbb{R}^{N}} u(x)^{\frac{p+q}{2}} I_{\beta}(x-y) u(y)^{\frac{p+q}{2}} d x d y\right)^{2},
\end{aligned}
$$

so that

$$
\int_{\mathbb{R}^{N}}\left(I_{\beta} * u^{p}\right) u^{q} \geq \iint_{\mathbb{R}^{N} \times \mathbb{R}^{N}} u(x)^{\frac{p+q}{2}} I_{\beta}(x-y) u(y)^{\frac{p+q}{2}} .
$$

Using the lower bound Eq. 14 and the fact that $\frac{p+q}{2}=\frac{N+\beta}{2(N-\alpha)}>0$ we deduce

$$
\begin{aligned}
\int_{\mathbb{R}^{N}}\left(I_{\beta} * u^{p}\right) u^{q} & \geq \int_{\mathbb{R}^{N} \backslash B_{1}} \int_{\mathbb{R}^{N} \backslash B_{1}} u(x)^{\frac{p+q}{2}} I_{\beta}(x-y) u(y)^{\frac{p+q}{2}} d x d y \\
& \geq c \int_{\mathbb{R}^{N} \backslash B_{1}} \int_{\mathbb{R}^{N} \backslash B_{1}} \frac{1}{|x|^{\frac{N+\beta}{2}}} I_{\beta}(x-y) \frac{1}{|y|^{\frac{N+\beta}{2}}} d x d y=\infty .
\end{aligned}
$$

Hence,

$$
\lim _{R \rightarrow \infty} \int_{B_{R}}\left(I_{\beta} * u^{p}\right) u^{q}=\infty .
$$

Since $u$ satisfies Eq. 1, from Lemma 2.2 with $\theta=\frac{p+q-1}{4}=\frac{\alpha+\beta}{4(N-\alpha)}$ and $f=\left(I_{\beta} * u^{p}\right) u^{q}$ we find

$$
\int_{B_{2 R} \backslash B_{R}} u^{\frac{p+q-1}{4}} \geq \int_{B_{2 R} \backslash B_{R}}\left(I_{\alpha} * f\right)^{\frac{p+q-1}{4}} \geq C R^{N-\frac{\alpha+\beta}{4}}\left(\int_{B_{R}}\left(I_{\beta} * u^{p}\right) u^{q}\right)^{\frac{p+q-1}{4}} .
$$

From the above estimate and Eq. 25 we deduce

$$
\lim _{R \rightarrow \infty} \frac{1}{R^{N-\frac{\alpha+\beta}{4}}} \int_{B_{2 R} \backslash B_{R}} u^{\frac{p+q-1}{4}}=\infty,
$$

which contradicts the upper bound in Eq. 24.

If $\alpha+\beta \geq N$ we give precise lower bounds on $\int_{B_{2 R} \backslash B_{R}} u^{q-1}$ to obtain a further nonexistence result.

Proposition 4.3 Let $p, q>0$ and assume that $\alpha+\beta>N$ and $1<q \leq \frac{\beta}{N-\alpha}$. If $u \geq 0$ is a solution of Eq. 1 then $u \equiv 0$.

Proof Assume that $u>0$ on a set of positive measure and let $R>1$ be large. From Eq. 14 we obtain

$$
\left(\int_{B_{2 R} \backslash B_{R}} u^{\frac{q-1}{2}}\right)^{2} \geq c R^{2 N-(N-\alpha)(q-1)} .
$$

On the other hand, by Corollary 3.4,

$$
\left(\int_{B_{2 R} \backslash B_{R}} u^{\frac{q-1}{2}}\right)^{2} \leq \frac{C R^{3 N-\beta-\alpha}}{\int_{B_{2 R}} u^{p}} \leq C^{\prime} R^{3 N-\beta-\alpha} .
$$

Now Eqs. 26 and 27 yield a contradiction if $q<\frac{\beta}{N-\alpha}$. 
In the critical case $q=\frac{\beta}{N-\alpha}$ we use Eq. 11 to find

$$
\left(I_{\beta} * u^{p}\right)(x) \geq \frac{c}{|x|^{N-\beta}} \int_{B_{1}} u^{p} \quad \text { for all } x \in B_{R} \backslash B_{1} .
$$

Combining this estimate with Eq. 14 we obtain

$$
\begin{aligned}
\int_{B_{R}}\left(I_{\beta} * u^{p}\right) u^{q} & \geq c\left(\int_{B_{1}} u^{p}\right) \int_{B_{R} \backslash B_{1}} \frac{u(x)^{q}}{|x|^{N-\beta}} d x \\
& \geq c^{\prime} \int_{B_{R} \backslash B_{1}} \frac{1}{|x|^{N-\beta+(N-\alpha) q}} d x=c^{\prime \prime} \log (R),
\end{aligned}
$$

since $(N-\alpha) q=\beta$. By Lemma 2.2 with $\theta=\frac{q-1}{2}>0$,

$$
\begin{aligned}
\int_{B_{2 R} \backslash B_{R}} u^{\frac{q-1}{2}} & \geq \int_{B_{2 R} \backslash B_{R}}\left(I_{\alpha} *\left(\left(I_{\beta} * u^{p}\right) u^{q}\right)\right)^{\frac{q-1}{2}} \\
& \geq c R^{N-(N-\alpha) \frac{q-1}{2}}\left(\int_{B_{R}}\left(I_{\beta} * u^{p}\right) u^{q}\right)^{\frac{q-1}{2}} \\
& \geq c^{\prime} R^{\frac{2 N-(N-\alpha)(q-1)}{2}} \log \frac{q-1}{2}(R) \\
& =c^{\prime} R^{\frac{3 N-\alpha-\beta}{2}} \log ^{\frac{q-1}{2}}(R),
\end{aligned}
$$

which contradicts Eq. 27.

The transitional locally linear case $q=1$ requires a separate consideration.

Proposition 4.4 Let $p>0$ and assume that $\alpha+\beta>N$ and $q=1$. If $u \geq 0$ is a solution of Eq. 1 then $u \equiv 0$.

Proof Using Corollary 3.4, for any $R>0$ we have

$$
R^{3 N-\alpha-\beta} \geq c\left(\int_{B_{R}} u^{p}\right)\left(\int_{B_{2 R} \backslash B_{R}} 1\right)^{2}=c R^{2 N}\left(\int_{B_{R}} u^{p}\right) .
$$

Since $\alpha+\beta>N$, it follows that $u \equiv 0$.

In the sublinear case $q<1$ we deduce an additional restriction on the admissible range of the exponent $q$.

Proposition 4.5 Let $p, q>0$ and assume that $p+q \geq 1, q<1$ and

$$
q \leq 1-\frac{N-\alpha-\beta}{N} p .
$$

If $u \geq 0$ is a solution of Eq. 1 then $u \equiv 0$. 
Proof Since $q<1$, by Hölder's inequality we deduce

$$
\begin{aligned}
c R^{N}=\int_{B_{2 R} \backslash B_{R}} 1 & =\int_{B_{2 R} \backslash B_{R}} u^{p \frac{1-q}{2 p+1-q}} u^{\frac{q-1}{2} \frac{2 p}{2 p+1-q}} \\
& \leq\left(\int_{B_{2 R} \backslash B_{R}} u^{p}\right)^{\frac{1-q}{2 p+1-q}}\left(\int_{B_{2 R} \backslash B_{R}} u^{\frac{q-1}{2}}\right)^{\frac{2 p}{2 p+1-q}} \\
& =\left[\left(\int_{B_{2 R} \backslash B_{R}} u^{p}\right)\left(\int_{B_{2 R} \backslash B_{R}} u^{\frac{q-1}{2}}\right)^{2}\right]^{\frac{1-q}{2 p+1-q}}\left(\int_{B_{2 R} \backslash B_{R}} u^{\frac{q-1}{2}}\right)^{2 \frac{p+q-1}{2 p+1-q}} .
\end{aligned}
$$

By Corollary 3.4 we have

$$
\left(\int_{B_{2 R}} u^{p}\right)\left(\int_{B_{2 R} \backslash B_{R}} u^{\frac{q-1}{2}}\right)^{2} \leq C R^{3 N-\alpha-\beta},
$$

which yields

$$
\left(\int_{B_{2 R} \backslash B_{R}} u^{p}\right)\left(\int_{B_{2 R} \backslash B_{R}} u^{\frac{q-1}{2}}\right)^{2} \leq C R^{3 N-\alpha-\beta},
$$

and on the other hand

$$
\left(\int_{B_{2 R} \backslash B_{R}} u^{\frac{q-1}{2}}\right)^{2} \leq C \frac{R^{3 N-\beta-\alpha}}{\int_{B_{2 R}} u^{p}} \leq C^{\prime} R^{3 N-\alpha-\beta} .
$$

If $q<1-\frac{N-\beta-\alpha}{N} p$, we use Eqs. 30- 31 in 29 to raise a contradiction since $p+q \geq 1$. If $q=1-\frac{N-\beta-\alpha}{N} p$, we use Eq. 31 and Hölder's inequality to further estimate

$$
\int_{B_{2 R} \backslash B_{R}} u^{p} \geq \frac{\left(\int_{B_{2 R} \backslash B_{R}} 1\right)^{1+\frac{2 p}{1-q}}}{\left(\int_{B_{2 R} \backslash B_{R}} u^{\frac{q-1}{2}}\right)^{\frac{2 p}{1-q}}} \geq c R^{N-\frac{p}{1-q}(N-\alpha-\beta)}=c .
$$

This shows that $u^{p} \notin L^{1}\left(\mathbb{R}^{N}\right)$ and

$$
\lim _{R \rightarrow \infty} \int_{B_{2 R}} u^{p}=\infty
$$

Using this fact in Eq. 31 we deduce

$$
\left(\int_{B_{2 R} \backslash B_{R}} u^{\frac{q-1}{2}}\right)^{2}=o\left(R^{3 N-\alpha-\beta}\right) \quad \text { as } R \rightarrow \infty .
$$

We now use this last estimate and Eqs. 30 in 29 to conclude.

\section{Optimal Decay and Existence}

If $u \geq 0$ is a solution of Eq. 1 , then either $u \equiv 0$ or $u$ must obey the "natural" lower bound Eq. 14, which implies in particular, the integral lower bound

$$
\int_{B_{2 R} \backslash B_{R}} u \geq c R^{\alpha}
$$

In the region $q<1$ the estimate Eq. 22 of Corollary 3.4 leads to an integral lower bound which improves upon Eq. 32 when $\alpha+\beta<N$ and $q<\frac{\beta}{N-\alpha}$. 
Proposition 5.1 Let $p, q>0$ and assume that $\alpha+\beta<N$ and $q<\frac{\beta}{N-\alpha}<1$. If $u \geq 0$ is a solution of Eq. 1, then either $u \equiv 0$ or

$$
\int_{B_{2 R} \backslash B_{R}} u \geq c R^{\frac{\alpha+\beta-N q}{1-q}} .
$$

As pointed out in Remark 1.2, since $\alpha+\beta<N$ and $q<\frac{\beta}{N-\alpha}<1$, the exponent of $R$ in Eq. 33 is greater than $\alpha$.

Proof From Corollary 3.4 we have

$$
\left(\int_{B_{2 R} \backslash B_{R}} u^{-\frac{1-q}{2}}\right)^{2} \leq c \frac{R^{3 N-\alpha-\beta}}{\int_{B_{2 R}} u^{p}} \leq c^{\prime} R^{3 N-\alpha-\beta} .
$$

Further, by Hölder's inequality (since $0<q<1$ ) we have

$$
\left(\int_{B_{2 R} \backslash B_{R}} u^{-\frac{1-q}{2}}\right)^{2} \geq\left(\int_{B_{2 R} \backslash B_{R}} u\right)^{-(1-q)}\left(\int_{B_{2 R} \backslash B_{R}} 1\right)^{3-q} .
$$

Now, the above estimates yield

$$
\left(\int_{B_{2 R} \backslash B_{R}} u\right)^{-(1-q)} \leq R^{N q-\alpha-\beta},
$$

which leads to Eq. 33 .

Our next step is to construct explicit solutions with the decay which match or near-match the lower bounds in Eq. 32 and Eq. 33. Before we do this, we recall the following simple estimates, cf. [15, Lemma A.1 and A.2] which are frequently used in the proofs below.

Lemma 5.2 Let $v \in L_{l o c}^{1}\left(\mathbb{R}^{N}\right), \gamma \in(0, N)$ and $s>\gamma$. If

$$
\limsup _{|x| \rightarrow \infty} v(x)|x|^{s}<\infty,
$$

then

$$
\begin{aligned}
& \limsup _{|x| \rightarrow \infty}\left(I_{\gamma} * v\right)(x)|x|^{s-\gamma}<\infty \text { if } \gamma<s<N, \\
& \limsup _{|x| \rightarrow \infty}\left(I_{\gamma} * v\right)(x) \frac{|x|^{N-\gamma}}{\log |x|}<\infty \text { if } s=N, \\
& \limsup _{|x| \rightarrow \infty}\left(I_{\gamma} * v\right)(x)|x|^{N-\gamma}<\infty \text { if } s>N .
\end{aligned}
$$

Lemma 5.3 Let $v \in L_{l o c}^{1}\left(\mathbb{R}^{N}\right), \gamma \in(0, N)$ and $\sigma \in \mathbb{R}$. If

$$
\limsup _{|x| \rightarrow \infty} v(x) \frac{|x|^{N}}{(\log |x|)^{\sigma}}<\infty
$$


then

$$
\begin{array}{r}
\limsup _{|x| \rightarrow \infty}\left(I_{\gamma} * v\right)(x)|x|^{N-\gamma}<\infty \text { if } \sigma<-1, \\
\limsup _{|x| \rightarrow \infty}\left(I_{\gamma} * v\right)(x) \frac{|x|^{N-\gamma}}{(\log (\log |x|))}<\infty \text { if } \sigma=-1, \\
\limsup _{|x| \rightarrow \infty}\left(I_{\gamma} * v\right)(x) \frac{|x|^{N-\gamma}}{(\log |x|)^{\sigma+1}}<\infty \text { if } \sigma>-1 .
\end{array}
$$

Proposition 5.4 Assume that

$$
p>\frac{\beta}{N-\alpha}, \quad p+q>\frac{N+\beta}{N-\alpha} \quad \text { and } \quad q>\frac{\beta}{N-\alpha} .
$$

Then, Eq. 1 admits a positive radial solution $u \in C\left(\mathbb{R}^{N}\right)$ which satisfies

$$
\limsup _{|x| \rightarrow \infty} u(x)|x|^{N-\alpha}<\infty .
$$

Proof Let $0<\varepsilon<q(N-\alpha)-\beta$ and take $u(x)=(1+|x|)^{-(N-\alpha)}$. Since $p(N-\alpha)>\beta$ we can apply the estimates in Lemma 5.2 to deduce

$$
\left(I_{\beta} * u^{p}\right)(x) \leq c_{1}\left\{\begin{array}{ll}
(1+|x|)^{\beta-p(N-\alpha)} & \text { if } p(N-\alpha)<N \\
(1+|x|)^{\beta-N} & \text { if } p(N-\alpha)>N \\
(1+|x|)^{\beta-N} \log (|x|+e) & \text { if } p(N-\alpha)=N
\end{array} \quad \text { in } \mathbb{R}^{N},\right.
$$

for some constant $c_{1}>0$. Thus,

$$
\left[\left(I_{\beta} * u^{p}\right) u^{q}\right](x) \leq c_{1}\left\{\begin{array}{ll}
(1+|x|)^{\beta-(p+q)(N-\alpha)} & \text { if } p(N-\alpha)<N \\
(1+|x|)^{\beta-N-q(N-\alpha)} & \text { if } p(N-\alpha)>N \\
(1+|x|)^{\beta-N-q(N-\alpha)} \log (|x|+e) & \text { if } p(N-\alpha)=N
\end{array} \quad \text { in } \mathbb{R}^{N} .\right.
$$

In particular, one may further estimate as

$$
\left[\left(I_{\beta} * u^{p}\right) u^{q}\right](x) \leq c_{1}\left\{\begin{array}{ll}
(1+|x|)^{\beta-(p+q)(N-\alpha)} & \text { if } p(N-\alpha)<N \\
(1+|x|)^{\beta-N-q(N-\alpha)+\varepsilon} & \text { if } p(N-\alpha) \geq N
\end{array} \quad \text { in } \mathbb{R}^{N} .\right.
$$

Since $(p+q)(N-\alpha)-\beta>N$ and $N-\beta+q(N-\alpha)-\varepsilon>N$ it follows from the third estimate in Lemma 5.2 that

$$
I_{\alpha} *\left[\left(I_{\beta} * u^{p}\right) u^{q}\right](x) \leq c_{2}(1+|x|)^{\alpha-N}=c_{2} u(x) \text { in } \mathbb{R}^{N},
$$

where $c_{2}>0$ is a constant. Thus, the continuous function $U(x)=c_{2}^{-1 /(p+q-1)} u(x)$ is a solution of Eq. 1 which satisfies Eq. 35 .

Proposition 5.5 Assume that

$$
1-\frac{N-\alpha-\beta}{N} p<q<\frac{\beta}{N-\alpha}<1 .
$$

Then, Eq. 1 admits a positive radial solution $u \in C\left(\mathbb{R}^{N}\right)$ which satisfies

$$
\limsup _{|x| \rightarrow \infty} u(x)|x|^{\frac{N-\alpha-\beta}{1-q}}<\infty .
$$


Proof Let $u(x)=(1+|x|)^{-k}$ where $k=(N-\alpha-\beta) /(1-q)$. Since $1-(N-\alpha-\beta) p / N<$ $q$, we have $p k>N$, and hence by the third estimate of Lemma 5.2 we have

$$
I_{\beta} * u^{p} \leq c_{1}(1+|x|)^{\beta-N} \text { in } \mathbb{R}^{N},
$$

for some constant $c_{1}>0$. Since $\beta-N-k q=-\frac{N-\beta-\alpha q}{1-q}$, we have

$$
\left(I_{\beta} * u^{p}\right) u^{q} \leq c_{2}(1+|x|)^{-\frac{N-\beta-\alpha q}{1-q}} \text { in } \mathbb{R}^{N} .
$$

Since $q<\frac{\beta}{N-\alpha}<1$, we have $\alpha<\frac{N-\beta-\alpha q}{1-q}<N$. Hence, by the first estimate of Lemma 5.2 we have

$$
I_{\alpha} *\left[\left(I_{\beta} * u^{p}\right) u^{q}\right] \leq c_{2}(1+|x|)^{-k} \quad \text { in } \mathbb{R}^{N},
$$

where $c_{2}>0$ is a constant. Thus, $U(x)=c_{2}^{-1 /(p+q-1)}(1+|x|)^{-k}$ is a continuous solution of Eq. 1. Moreover,

$$
\limsup _{|x| \rightarrow \infty} U(x)|x|^{k}<\infty
$$

Proposition 5.6 Assume that

$$
\alpha+\beta=N, \quad p>\frac{N}{N-\alpha} \text { and } q=1 .
$$

Then, for every $m>0$ inequality Eq. 1 admits a positive radial solution $u \in C\left(\mathbb{R}^{N}\right)$ which satisfies

$$
\limsup _{|x| \rightarrow \infty} u(x)|x|^{N-\alpha-m}<\infty
$$

Proof Let $m>0$. Since $p>\frac{N}{N-\alpha}=\frac{N}{\beta}$, we see that $\beta-\frac{N}{p}>0$. Set

$$
\delta=\left\{\begin{array}{ll}
m & \text { if } 0<m<\beta-\frac{N}{p}, \\
\frac{1}{2}\left(\beta-\frac{N}{p}\right) & \text { if } m \geq \beta-\frac{N}{p},
\end{array} \quad \text { and } \quad k=\beta-\delta .\right.
$$

Then,

$$
k p= \begin{cases}(\beta-m) p>N & \text { if } 0<m<\beta-\frac{N}{p}, \\ \frac{\beta p}{2}+\frac{N}{2}>N & \text { if } m \geq \beta-\frac{N}{p} .\end{cases}
$$

Let $u(x)=(1+|x|)^{-k}$. By the third estimate of Lemma 5.2 we see that $I_{\beta} * u^{p} \leq c_{1}(1+$ $|x|)^{\beta-N}$ in $\mathbb{R}^{N}$ for some constant $c_{1}>0$, and hence

$$
\left(I_{\beta} * u^{p}\right) u^{q} \leq c_{1}(1+|x|)^{\beta-N-k q} \quad \text { in } \mathbb{R}^{N} .
$$

Since $\alpha<-\beta+N+k q<N$, by the first estimate of Lemma 5.2 we see that

$$
I_{\alpha} *\left[\left(I_{\beta} * u^{p}\right) u^{q}\right] \leq c_{2}(1+|x|)^{\alpha+\beta-N-k q} \quad \text { in } \mathbb{R}^{N},
$$

for some constant $c_{2}>0$. Since $\alpha+\beta-N-k q=-k$, we have

$$
I_{\alpha} *\left[\left(I_{\beta} * u^{p}\right) u^{q}\right] \leq c_{2} u \quad \text { in } \mathbb{R}^{N} .
$$

Thus, $U(x)=c_{2}^{-1 /(p+q-1)}(1+|x|)^{-k}$ is a continuous solution of Eq. 1. Moreover,

$$
\limsup _{|x| \rightarrow \infty} U(x)|x|^{N-\alpha-m} \leq \limsup _{|x| \rightarrow \infty} U(x)|x|^{k}<\infty .
$$




\section{Proposition 5.7 Assume that}

$$
p>\frac{N}{N-\alpha} \quad \text { and } \quad q=\frac{\beta}{N-\alpha}<1 .
$$

Then, for $m \geq \frac{N-\alpha}{N-\alpha-\beta}$ inequality Eq. 1 admits a positive radial solution $u \in C\left(\mathbb{R}^{N}\right)$, which satisfies

$$
\limsup _{|x| \rightarrow \infty} u(x)|x|^{N-\alpha}(\log |x|)^{-m}<\infty .
$$

Proof Take

$$
u(x)=(1+|x|)^{-(N-\alpha)}(\log (e+|x|))^{m} .
$$

Since $-(N-\alpha) p<-N$, by the third estimate of Lemma 5.2 we see that $I_{\beta} * u^{p} \leq$ $c_{1}(1+|x|)^{\beta-N}$ for some constant $c_{1}>0$, and hence

$$
\left(I_{\beta} * u^{p}\right) u^{q} \leq c_{1}(1+|x|)^{\beta-N-(N-\alpha) q}(\log (e+|x|))^{m q}=c_{1}(1+|x|)^{-N}(\log (e+|x|))^{m q} .
$$

By the third estimate of Lemma 5.3 we see that

$$
I_{\alpha} *\left[\left(I_{\beta} * u^{p}\right) u^{q}\right] \leq c_{2}(1+|x|)^{-(N-\alpha)}(\log (e+|x|))^{m q+1} \quad \text { in } \mathbb{R}^{N},
$$

for some constant $c_{2}>0$. Since $m \geq \frac{N-\alpha}{N-\alpha-\beta}$, we have

$$
I_{\alpha} *\left[\left(I_{\beta} * u^{p}\right) u^{q}\right] \leq c_{2} u .
$$

Thus, $U(x)=c_{2}^{-1 /(p+q-1)} u$ is a continuous solution of Eq. 1 .

Funding Open Access funding provided by the IReL Consortium

Data Availability Data sharing not applicable to this article as no datasets were generated or analysed during the current study.

Open Access This article is licensed under a Creative Commons Attribution 4.0 International License, which permits use, sharing, adaptation, distribution and reproduction in any medium or format, as long as you give appropriate credit to the original author(s) and the source, provide a link to the Creative Commons licence, and indicate if changes were made. The images or other third party material in this article are included in the article's Creative Commons licence, unless indicated otherwise in a credit line to the material. If material is not included in the article's Creative Commons licence and your intended use is not permitted by statutory regulation or exceeds the permitted use, you will need to obtain permission directly from the copyright holder. To view a copy of this licence, visit http://creativecommons.org/licenses/by/4.0/.

\section{References}

1. Abatangelo, N., Jarohs, S., Saldaña, A.: Positive powers of the Laplacian: from hypersingular integrals to boundary value problems. Commun. Pure Appl. Anal. 17, 899-922 (2018)

2. Caristi, G., D’Ambrosio, L., Mitidieri, E.: Representation formulae for solutions to some classes of higher order systems and related Liouville theorems. Milan J. Math. 76, 27-67 (2008)

3. Chen, W., Li, C., Ou, B.: Qualitative properties of solutions for an integral equation. Discrete Contin. Dyn. Syst. 12, 347-354 (2005)

4. Chen, W., Li, C., Ou, B.: Classification of solutions for an integral equation. Comm. Pure Appl. Math. 59, 330-343 (2006)

5. Froöhlich, H.: Theory of electrical breakdown in ionic crystal. Proc. Roy. Soc. Ser. A 160, 230-241 (1937)

6. Froöhlich, H.: Electrons in lattice fields. Adv. Phys. 3, 325-361 (1954) 
7. Ghergu, M., Miyamoto, Y., Moroz, V.: Polyharmonic inequalities with nonlocal terms. J. Diff. Equ. 296, 799-821 (2021)

8. Ghergu, M., Taliaferro, S.: Asymptotic behavior at isolated singularities for solutions of nonlocal semilinear elliptic systems of inequalities, Calc. Var. Partial Diff. Equ. 54, 1243-1273 (2015)

9. Landkof, N.S.: Foundations of modern potential theory. Die Grundlehren der mathematischen Wissenschaften, Band 180, p. x+424. Springer, New York (1972)

10. Le, P.: Liouville theorems for an integral equation of Choquard type. Commun. Pure Appl. Anal. 19, 771-783 (2020)

11. Lei, Y.: Asymptotic properties of positive solutions of the Hardy-Sobolev type equations. J. Diff. Equ. 254, 1774-1799 (2013)

12. Lei, Y.: Qualitative analysis for the static Hartree-type equations. SIAM J. Math. Anal. 45, 388-406 (2013)

13. Lieb, E.H.: Existence and uniqueness of the minimizing solution of Choquard's nonlinear equation. Stud. Appl. Math. 57, 93-105 (1976/77)

14. Moroz, V., Van Schaftingen, J.: Nonlocal Hardy type inequalities with optimal constants and remainder terms. Ann. Univ. Buchar. Math. Ser. 3(LXI), 187-200 (2012)

15. Moroz, V., Van Schaftingen, J.: Nonexistence and optimal decay of supersolutions to Choquard equations in exterior domains. J. Diff. Equ. 254, 3089-3145 (2013)

16. Moroz, V., Van Schaftingen, J.: A guide to the Choquard equation. J. Fixed Point Theory Appl. 19, 773-813 (2017)

17. Pekar, S.: Untersuchung über Die Elektronentheorie Der Kristalle. Akademie, Berlin (1954)

18. Stein, E.M.: Singular integrals and differentiability properties of functions, p. xiv+290. Princeton University Press, Princeton (1970)

19. Villavert, J.: Asymptotic and optimal Liouville properties for Wolff type integral systems. Nonlinear Anal. 130, 102-120 (2016)

20. Villavert, J.: Qualitative properties of solutions for an integral system related to the Hardy-Sobolev inequality. J. Diff. Equ. 258, 1685-1714 (2015)

Publisher's Note Springer Nature remains neutral with regard to jurisdictional claims in published maps and institutional affiliations. 